\title{
KPZ Formulas for Weyl-Invariant Induced Gravity and Topologically Massive Gravity *
}

\author{
Giovanni Amelino-Camelia ${ }^{a}$ \\ ${ }^{a}$ Theoretical Physics, University of Oxford \\ 1 Keble Road, Oxford OX1 3NP, U.K.
}

I discuss the applicability in Weyl-invariant induced gravity and topologically massive gravity of certain formulas originally derived by Knizhnik, Polyakov, and Zamolodchikov in the context of diffeomorphism-invariant induced gravity.

Over the last ten years there has been a strong interest in the investigation of lowdimensional quantum gravity toy models. A very popular such model is the two-dimensional (2D) "induced" gravity characterized by a diffeomorphism-invariant measure [1] for the functional (path) integration over the metric tensor field and the action

$$
\begin{aligned}
S(g)=\frac{d}{96 \pi} \int d^{2} \xi_{1} d^{2} \xi_{2} \sqrt{g\left(\xi_{1}\right)} R\left(g\left(\xi_{1}\right)\right) \\
\cdot \square^{-1}\left(\xi_{1}, \xi_{2}\right) \sqrt{g\left(\xi_{2}\right)} R\left(g\left(\xi_{2}\right)\right),
\end{aligned}
$$

where $g$ is the metric tensor, $\sqrt{g}$ is the square root of the determinant of $g, \square^{-1}$ is the inverse of the Laplace-Beltrami operator, and, for simplicity, I set the cosmological constant to zero. This can be seen as the quantum gravity induced by integrating out, with a diffeomorphism-invariant path-integral measure [1], the "matter" degrees of freedom of the field theory with action

$\mathcal{I}(X, g)=\frac{1}{2} \int d^{2} \xi \sqrt{g} g^{\mu \nu} \partial_{\mu} X^{A} \partial_{\nu} X^{A}$,

where the $X^{A}$, with $A=1,2, \ldots, d$, denote the $d$ components of the massless "matter" scalar field. Note that the action (2) is invariant under both diffeomorphisms and Weyl transformations, while

\footnotetext{
*Talk given at the 2nd Topical Meeting on Constrained Dynamics and Quantum Gravity, Santa Margherita, Ligure, Italy, 17-21 September, 1996. To be published in the proceedings (Editor: V. de Alfaro).

OUTP-96-72P

hep-th/9701161

December 1996
}

(1) is only diffeomorphism-invariant. This reflects the presence of an anomaly, i.e. the fact that there are no path-integral measures with invariance under both diffeomorphisms and Weyl transformations [1,2].

A well-appreciated result, first derived by Knizhnik, Polyakov, and Zamolodchikov 11, concerns the Green's functions of diffeomorphisminvariant induced gravity. It is encoded in the following anomalous Ward identities of the lightcone-gauge formulation of the theory (in which the entire physical content of the metric tensor resides in its $g_{++}$component):

$$
\begin{aligned}
\sum_{i}^{n} & \left\langle g_{++}\left(\xi_{1}\right) \ldots \delta_{\delta f} g_{++}\left(\xi_{i}\right) \ldots g_{++}\left(\xi_{n}\right)\right\rangle \\
= & \frac{28-d-\lambda}{i 48 \pi} \int d \xi^{2} \delta f(\xi) \\
& \cdot\left\langle\partial_{-}^{3} g_{++}(\xi) g_{++}\left(\xi_{1}\right) \ldots g_{++}\left(\xi_{n}\right)\right\rangle,
\end{aligned}
$$

where $\delta_{\delta f}$ is such that

$\delta_{\delta f} \chi=\left(2 \partial_{+}-\chi \partial_{-}\right) \delta f+\delta f \partial_{-} \chi$

and $\lambda$ is a constant characterizing the measure 1, 2 that can be expressed in terms of the number of components of the massless "matter" scalar field using the relation [1]

$\lambda=\frac{43-d-\sqrt{(d-1)(d-25)}}{2}$.

Another important observation due to Knizhnik, Polyakov, and Zamolodchikov [1] is frequently exploited in the conformal-gauge formulation of the theory, which is Liouville theory. It 
establishes the scaling relations [1] for primary fields of Liouville theory

$\hat{\Delta}-\hat{\Delta}_{0}=\frac{\hat{\Delta}(1-\hat{\Delta})}{c+2}$

where $c$ is the central charge of the $S L(2, R)$ current algebra, $\hat{\Delta}_{0}$ is the bare conformal dimension of the primary field, and $\hat{\Delta}$ is its Liouville-dressed conformal dimension.

Although these results were originally derived in diffeomorphism-invariant induced gravity, it has been recently realized that they have wider applicability. Let us start by considering Weylinvariant induced gravity [3, 4, 2, 5, 5, which is the 2D quantum gravity induced by integrating out, with a Weyl-invariant path-integral measure [4:2], the "matter" degrees of freedom of the field theory with action (21). The action governing the dynamics of the metric tensor field is

$$
\begin{aligned}
\mathcal{S}(\gamma)=\frac{d}{96 \pi} \int d^{2} \xi_{1} d^{2} \xi_{2} R\left(\gamma\left(\xi_{1}\right)\right) \\
\cdot \square^{-1}\left(\xi_{1}, \xi_{2}\right) R\left(\gamma\left(\xi_{2}\right)\right),
\end{aligned}
$$

where I used the short-hand notation $\gamma \equiv \sqrt{g} g$. A Weyl-invariant measure for the functional (path) integration over the metric tensor field $g$, depending on $g$ only through $\gamma$, has also been identified [3, 4, 2]. The fact that both the measure and (बi) depend on $g$ only through $\gamma$ encodes the fact that the theory is Weyl invariant and invariant under diffeomorphisms of unit Jacobian (also called "S-diffeomorphisms" or "area-preserving diffeomorphisms" [3,4,2), while the theory is not fully diffeomorphism-invariant. Concerning the Green's functions of this Weyl-invariant induced gravity it has been recently realized [2] that in an appropriate (light-cone-like) gauge the following relations hold

$$
\begin{aligned}
& \sum_{i}^{n}\left\langle\gamma_{++}\left(\xi_{1}\right) \ldots \delta_{\delta f} \gamma_{++}\left(\xi_{i}\right) \ldots \gamma_{++}\left(\xi_{n}\right)\right\rangle \\
& =\frac{28-d-\lambda^{W}}{i 48 \pi} \int d \xi^{2} \delta f(\xi) \\
& \quad \cdot\left\langle\partial_{-}^{3} \gamma_{++}(\xi) \gamma_{++}\left(\xi_{1}\right) \ldots \gamma_{++}\left(\xi_{n}\right)\right\rangle,
\end{aligned}
$$

where $\lambda^{W}$ is a constant characterizing the Weyl- invariant measure and I denoted with $\gamma_{++}$the component of $\gamma$ that is relevant in the given gauge [2]. The correspondence between (3) and (8) describes the applicability of the KPZ formula (3) in the context of Weyl-invariant induced gravity.

Actually, it has been realized in Ref. [6] that a similar argument applies also to other 2D induced-gravity theories. In particular, a oneparameter family of such theories, all invariant under diffeomorphisms of unit Jacobian but not fully diffeomorphism-invariant, has been identified, and evidence has been found [6] in support of the applicability of the KPZ formula (3) in those theories. I concentrate here on the special Weylinvariant case for simplicity.

Let us now move on to a 3D context and consider a theory in which topologically massive gravity is coupled to topologically massive electrodynamics and a charged massive scalar field. It has been recently realized [7] that the KPZ formula (6) encodes (in the sense that I clarify in the following) the relation between the AharonovBohm amplitude of such a theory and the ordinary Aharonov-Bohm amplitude [8]. This is encoded formally in a property of the transmuted spin $\Delta$, which is defined as the overall coefficient of the Aharonov-Bohm amplitude [7]

$\Delta \frac{16 \pi}{i} \operatorname{dim}(\mathcal{G}) \frac{\epsilon_{\mu \nu \lambda} p_{1}^{\mu} p_{2}^{\nu} q^{\lambda}}{q^{2}}$,

where $p_{1}$ and $p_{2}$ are the initial momenta of the two charged particles involved in the scattering, $q$ is the momentum transfer, and $\mathcal{G}$ is the gauge group. For example, in the ordinary (abelian $\mathcal{G}$ and no gravitational degrees of freedom) Aharonov-Bohm amplitude the transmuted spin is given by the inverse of the Chern-Simons coefficient.

In Ref. [7] evidence is provided in support of the relation

$\Delta-\Delta_{0}=\frac{\Delta(\Delta-1)}{k^{\prime}+2}$

between the bare transmuted spin $\Delta_{0}$ (i.e. the value taken by the transmuted spin when the ordinary gauge degrees of freedom are present, but

${ }^{2}$ This constant $\lambda^{W}$ has been conjectured 2 to be equal to the constant $\lambda$ of the diffeomorphism-invariant case. 
the gravitational degrees of freedom are turned off), the gravitationally-dressed transmuted spin $\Delta$ (i.e. the value taken by the transmuted spin when also the gravitational degrees of freedom of topologically massive gravity are turned on), and the gravitational Chern-Simons coefficient $k^{\prime}$.

The formula (10) reproduces the KPZ formula (6) upon identifying the bare (respectively the Liouville-dressed) conformal dimensions of primary fields in Liouville theory with the bare (respectively the gravitationally-dressed) transmuted spin of topologically massive gravity, and assuming the relation $c=-k^{\prime}-4$ between the central charge $c$ of Liouville theory and the gravitational Chern-Simons coefficient $k^{\prime}$.

This observation provides one of the strongest pieces of evidence in support of a relation of the type discussed in Refs. [9.10] between topologically massive gravity and Liouville theory, and can be used to motivate a topological membrane approach to string theory [11], in which the string world-sheet is filled in and viewed as the boundary of a three-manifold.

Similarly, the applicability of KPZ formulas in non-diffeomorphism-invariant induced-gravity theories can be used as a probe of the relation among the different 2D induced gravity theories (diffeomorphism-invariant or not). In particular, it has been suggested [2,6] that the members of a large class of such theories might all be essentially equivalent (as originally established for diffeomorphism-invariant induced gravity) to Liouville theory. Interestingly, this has led to a different viewpoint [12,6, 13,14, on the phenomenon of Hawking radiation in 2D gravity. The presence of a (non-necessarily-Weyl) anomaly and the residual invariance under diffeomorphisms of unit Jacobian are now seen as the essential elements of this phenomenon, whereas in earlier interpretations the presence of a Weyl anomaly was often emphasized.

The recent results on 2D induced gravity theories have also sparked renewed interest in the issues associated to the quantization of anomalous field theories. In particular, effort has been devoted 13, 15 to the investigation of the physical implications of local counter-terms in anomalous field theories. While the mass emergent in the chiral Schwinger model 16 is known to depend on the coefficient of one such local counter-term (associated to a one-parameter family of chiral symmetry breaking measures), similar phenomena have not been identified in the context of bosonic induced-gravity theories, at least in the case of topologically-trivial two-manifolds (the only case seriously investigated in the related studies). However, some evidence of physical implications of local counter-terms has been found in the context of chiral 2D induced gravity [13].

\section{ACKNOWLEDGEMENTS}

This work is based on results obtained in Refs. [2, 6, 13] in collaboration with D. Bak, L. Griguolo, and D. Seminara and in Ref. [7] in collaboration with I.I. Kogan and R.J. Szabo. I would like to thank V. de Alfaro and all those involved in the organization of the very successful 2nd Topical Meeting on Constrained Dynamics and Quantum Gravity. I would also like to acknowledge very stimulating discussions with several participants to the Meeting, particularly M. Cadoni, S. Carlip, S. Deser, R. Jackiw, S. Sciuto, and G. Veneziano. This work was supported in part by PPARC and by the Foundation Stiftelsen Blanceflor Boncompagni-Ludovisi.

\section{REFERENCES}

1. A.M. Polyakov, Phys. Lett. B103 (1981) 207; Mod. Phys. Lett. A2 (1987) 893; V.G. Knizhnik, A.M. Polyakov and A. B. Zamolodchikov, ibid. A3 (1988) 819; F. David, ibid. A3 (1988) 1651; J. Distler and H. Kawai, Nucl. Phys. B321 (1989) 509; N.E. Mavromatos and J.L. Miramontes, Mod. Phys. Lett. A4 (1989) 1847; E. D'Hoker and P.S. Kurzepa, ibid. A5 (1990) 1411.

2. G. Amelino-Camelia, D. Bak, and D. Seminara, Phys. Lett. B354 (1995) 213.

3. D.R. Karakhanian, R.P. Manvelian, R.L. Mkrtchian, Phys. Lett. B329 (1994) 185.

4. R. Jackiw, Report No. hep-th/9501016.

5. Weyl invariance is discussed within a different formalism in M. Reuter, Report No. hepth/9612158, and references therein. 
6. G. Amelino-Camelia and D. Seminara, Class. Quant. Grav. 13 (1996) 881.

7. G. Amelino-Camelia, I.I. Kogan, and R.J. Szabo, Nucl. Phys. B480 (1996) 413.

8. Y. Aharonov and D. Bohm, Phys. Rev. 115 (1959) 485.

9. S. Carlip, Nucl. Phys. B362 (1991) 111.

10. S. Carlip and I.I. Kogan, Phys. Rev. Lett. 64 (1990) 148; ibid. 67 (1991) 3647; Mod. Phys. Lett. A6 (1991) 171; S. Carlip, Phys. Rev. D45 (1992) 3584; I.I. Kogan, Phys. Lett. B256 (1991) 369; I.I. Kogan, Nucl. Phys. B375 (1992) 362; M.C. Ashworth, Report No. hepth/9510192.

11. I.I. Kogan, Phys. Lett. B231 (1989) 377.

12. J. Navarro-Salas, M. Navarro, and C.F. Talavera, Phys. Lett. B356 (1995) 217; J. Cruz, J. Navarro-Salas, M. Navarro, and C.F. Talavera, Report No. hep-th/9606097.

13. G. Amelino-Camelia, L. Griguolo, and D. Seminara, Phys. Lett. B371 (1996) 41.

14. M. Cadoni, Report No. hep-th/9610201.

15. M. Martellini, M. Spreafico, and K. Yoshida, Phys. Rev. D54 (1996) 7664.

16. R. Jackiw and R. Rajaraman, Phys. Rev. Lett. 54 (1985) 1219; for a review see R. Jackiw in Quantum Mechanics of Fundamental Systems 1, C. Teitelboim ed. (Plenum, New York, 1988). 